\title{
Software shakes up schizophrenia diagnosis
}

Computer analysis of brain images can diagnose schizophrenia in patients, possibly even before symptoms arise, say researchers at the University of Pennsylvania in Philadelphia.

The technique, which is based on the ability of computers to tease out subtle differences between brain images, has split neuroscientists, with some questioning the value of the information produced.

But Ruben Gur and his colleagues are convinced it works. Earlier this year, they claimed that they could use the technique to detect whether individuals are lying or telling the truth (see Nature 437, 457; 2005). Now they have turned their attention to mental disorders.

They used magnetic resonance imaging (MRI) to scan the brains of 69 schizophrenia patients and 79 healthy controls. The images were analysed by computer to produce an algorithm that could tell the two groups apart. Rather than focusing on specific areas of the brain thought to be affected by the disorder, as has been tried in the past, they looked for subtle changes across the whole brain.

This type of approach has proved successful before - but only for images used to derive the algorithm. As soon as fresh images were introduced, the success rate plummeted. But this time the researchers say that they have overcome this problem and that they were able to classify new individuals as schizophrenic or healthy with $81 \%$ accuracy (C. Davatzikos et al. Arch. Gen. Psychiatry 62, 1218-1227; 2005).

Gur makes bold claims for the technique, saying that it is ready to be used alongside clinical histories and interviews to help diagnose schizophrenia. And, because many of the people studied were 18 years old and in the early stages of the disease, he believes that it might be possible to use brain imaging to diagnose the disease before dassic symptoms appear.

${ }^{\alpha}$ Now we can give a computer a picture of a person's brain and ask whether or not this person has schizophrenia," says Gur. "This should do for schizophrenia what the echocardiogram did for heart disease."

Other experts are much more cautious. ${ }^{\alpha}$ The results are interesting and promising, but need replication," says Philip McGuire, an expert in brain scanning and schizophrenia at the Institute of Psychiatry in London.

"Finding differences compared with healthy people is the easy part," says Chris Frith, who specializes in schizophrenia and imaging at University College London. What will be useful, he says, is a way to distinguish people with schizophrenia from those suffering from related problems such as mania or severe depression.

Gur and his team now plan to compare schizophrenics with other mentally ill people. They also want to compare young sufferers with their family members.

Jennifer Wild 